\title{
Hand Hygiene Compliance by the Health Care Staff in a Pediatric Hospital
}

\author{
Farideh Shiva ${ }^{1}$; Mansour Kamali ${ }^{2}$; Fariba Shirvani ${ }^{2}$; Shahnaz Armin ${ }^{1,}{ }^{*}$; Roxana Ghanaiei ${ }^{1}$; \\ Sedigheh Rafiei Tabatabaie ${ }^{1}$; Seyed Ali Reza Fahimzad ${ }^{1}$; Ahmad Reza Shamshiri ${ }^{3}$; Abdollah \\ Karimi $^{1}$
}

${ }_{1}^{1}$ Pediatric Infections Research Center, Mofid Children's Hospital, Shahid Beheshti University of Medical Sciences, Tehran, IR Iran

${ }^{2}$ Emam Hossien Hospital, Tehran, IR Iran

${ }^{3}$ Department of Community Oral Health, Dental Research Center, Dentistry Research Institute, School of Dentistry, Tehran University of Medical Sciences, Tehran, IR Iran

*Corresponding author: Shahnaz Armin, Pediatric Infections Research Center, Mofid Children's Hospital, Shahid Beheshti University of Medical Sciences, Tehran, IR Iran. Tel: +989121544084, Fax: +98-2122226941, E-mail:armin_sh2000@yahoo.com

Received: March 12, 2014; Revised: April 8, 2014; Accepted: April 9, 2014

\begin{abstract}
Background: Hospital acquired infections lead to significant morbidity and mortality in hospitalized patients. Proper implementation of hand hygiene by health care personnel (HCP) prevents nosocomial infections.

Objectives: To monitor hand hygiene compliance by the medical and nursing staff in different wards of a children's hospital.

Patients and Methods: Four wards were selected for surveillance. In observation periods of 20 minutes each, a trained Pediatric resident observed four patient beds and hand hygiene behavior of health care professionals, physicians (house staff) and nurses, before and after touching the patients. The contacts were classified as 'high-risk' if the person came in contact with body fluids or 'low-risk' if there was no such contact. Findings were documented on a predesigned Hand Hygiene Observation Tool.

Results: Overall, 510 patient contacts were observed and 1020 hand hygiene opportunities before and after patient contact were recorded. In total, 302 contacts (59.2\%) were low risk and 208 (40.7\%) were high risk. The HCP used disposable and surgical gloves before $87,(41.82 \%)$ high risk and 27, (8.94\%), low risk contacts; however, no hand washing or the use of alcohol hand rubs before contact was recorded by the observer stationed by the patients' bed-side. The observer could not ascertain if the HCP washed their hands before entering the $3-6$ bedded rooms.

Conclusions:Serious lack of adherence to standard protocols for hand hygiene by the HCP together with inappropriate glove use, calls for an urgent strategic planning to educate, train and motivate the health care personnel in all aspects of hand hygiene practices.
\end{abstract}

Keywords:Hand Hygiene; Delivery of Health Care; Cross Infection

\section{Background}

Health care personnel (HCP) have been trained to deliver the best care to patients, without causing harm; however, the fact remains that the delivery of health care is associated with safety hazards, especially nosocomial infections. These infections constitute a significant problem in hospital care leading to significant morbidity and mortality in hospitalized patients $(1,2)$. The estimated incidence of hospital acquired infections (HAI) varies between developed and developing countries; however figures of about 1.5 million patients experiencing nosocomial infections at any given time have been quoted by the World Health Organization (1). Burden of HAI increases significantly in poor resource settings and with high risk patients, such as those admitted in intensive care units, or neonatal wards $(1,2)$.

Health care personnel (HCP) have been recognized as the prime source for transferring pathogenic microorganisms between different patients via unclean hands.
HCP hands harbor resident microorganisms just under the stratum corneum, as well as transient flora that are readily transferred from one patient to the other $(1,3)$. In a study conducted in Iran, it was revealed that the rate of contamination of health workers' hands by bacterial, viral and/or fungal pathogens after patient care episodes may be as high as $73.1 \%$, furthermore, the majority of the pathogens isolated in that study can cause nosocomial infections (2). Since the mid-nineteenth century, it has been acknowledged that hand cleansing by HCP is one of the most effective measures for preventing nosocomial infections, and decreasing the morbidity and mortality associated with hospital care $(1,3,4)$. Hand hygiene incorporates any procedure that leads to hand cleansing; washing hands with soap and water removes transient flora, while using alcohol based hand rubs eliminates both transient and resident flora, but it does not clean obviously soiled hands $(1,5)$. 
Opportunities for implementing hand hygiene have been defined by the WHO as 'five moments': before touching the patient, before aseptic procedures, after body fluid exposure, after touching a patient, and after touching patient surroundings $(1,3,6)$.

Regular surveillance of hand hygiene implementation in health care settings, especially hospitals, is an essential component of infection control programs. Methods advocated for measuring adherence of HCP to hand hygiene include; automated monitoring equipment, product consumption, self-reporting, patient-reporting, and assessment through direct observation $(1,7)$.

\section{Objectives}

In this study, we aimed to monitor hand hygiene compliance by the medical house staff and nursing personnel in different wards of a children's hospital affiliated with a University of Medical Sciences in Tehran.

\section{Patients and Methods}

The infectious diseases department, emergency ward, gastrointestinal department, which had three sections (rheumatology, endocrinology and gastroenterology), and the pediatric intensive care unit (PICU), of a children's hospital were selected for observation of HCP's hand hygiene behavior. Trained observers were stationed by the patients' bed side to monitor hand hygiene practices and document the results on two information sheets, Form 1 and 2. Form 1 was designed to record hand hygiene facilities available in the wards, i.e. the number and accessibility of hand washing stations and alcohol hand rubs (AHR), type of water faucets (elbow operated, electronic), hand dryers and display of hand hygiene posters.

The second form (Form 2) was an information sheet designed in accordance with the hand hygiene observation tool devised by researchers at the Royal Free and University College Medical School, in the United King$\operatorname{dom}(8)$.

Data recorded on the information sheet was as follows: during a specific time slot of 20 minutes, the observer would first define the field of view, i.e. patients occupying a specific number of beds (3-4 beds in one session); then record all opportunities for hand hygiene by a health care professional, ( resident, intern, or nursing personnel) who approached the patient. The 'opportunities' were divided into two: before touching the patient, and after touching the patient. These opportunities could be a 'high-risk' contact if the HCP came in contact with the patient's body fluids; examples of high risk contact included; collecting blood, setting up an intravenous infusion, inserting a urinary catheter, endos- copy, etc. Contacts were classified as 'low-risk' contact if there was no contact with body fluids, open wounds, or other secretions/excretions, but the HCP touched either the patient or their immediate surroundings. Instances of low risk contact involved tasks like; patient examination, recording heart rate or blood pressure, echocardiography, changing the patients' clothes or bedding, etc.

Hand hygiene behavior by the HCP was classified into six actions: 1) Washing hands with soap and water; 2) Utilizing AHR; 3) Using disposable gloves; 4) Wearing sterile gloves; 5) Decontaminating hands and using gloves; 6) No action. We added the actions of donning disposable or sterile gloves to our information sheet, although glove use was not part of the original hand hygiene observation tool (8).

Hand hygiene compliance was calculated by the following formula:

Overall compliance, $\%=$ Number of soap + AHR behaviors $\times 100 /$ total hand hygiene opportunities

Using disposable or sterile gloves without washing hands with soap and water or using AHR was recorded, but it was not considered as hand hygiene compliance and therefore it was not counted in the formula. SPSS software version 16 was used for the statistical analysis.

\section{Results}

Facilities for the implementation of hand hygiene available in the emergency ward, pediatric intensive care unit, infectious diseases department and the gastroenterology ward, are depicted in Table 1 . The study team observed 510 patient contacts in these departments. A total of one-thousand and twenty hand hygiene opportunities were recorded (before and after patient contact). The highest number of records came from the infectious diseases ward; 302 contacts (59.2\%) were low risk, and 208 (40.7\%) were high risk (Table 2).

Observed contacts from the different HCP were as follows: nursing personnel 59\%, pediatric residents $19.4 \%$, medical students $15.5 \%$, and fellowship physicians $6.1 \%$. The number of hand washing events and/or use of alcohol based hand rubs before patient contact was zero. Four individuals used hand hygiene after removal of gloves following a high risk contact. Disposable gloves were used before 73 high risk and 14 low risk contacts, while sterile surgical gloves were used before 14 high risk and 2 low risk contacts. Overall, glove use was as follows: 87 (41.82\%) of high risk contact and 27 (8.94\%), low risk contacts. There was no significant difference between the different departments in hand hygiene adherence, before or after contact $(\mathrm{P}=0.2)$, disposable glove use, $(\mathrm{P}=$ $0.4)$, or surgical glove use $(P=0.09$, Table 3$)$. 
Shiva F et al.

Table 1. Facilities Available for the Implementation of Hand Hygiene in Different Departments a

\begin{tabular}{|c|c|c|c|c|c|c|c|c|}
\hline \multirow{2}{*}{$\begin{array}{l}\text { Ward } \\
\text { Facility Available }\end{array}$} & \multicolumn{2}{|c|}{ Infectious Diseases } & \multicolumn{2}{|c|}{$\begin{array}{l}\text { Gastrointestinal, Rheumatology } \\
\text { and Endocrinology Department }\end{array}$} & \multicolumn{2}{|c|}{ PICU } & \multicolumn{2}{|c|}{$\begin{array}{c}\text { Emergency } \\
\text { Ward }\end{array}$} \\
\hline & Yes & No & Yes & No & Yes & No & Yes & No \\
\hline Hand operated taps & $x$ & & $x$ & & $x$ & & $x$ & \\
\hline Elbow operated taps & & $x$ & & $x$ & & $x$ & & $x$ \\
\hline Electronic taps & & $x$ & & $x$ & & $x$ & & $x$ \\
\hline Paper towels for drying hands & & $x$ & & $x$ & & $x$ & $\times$ & \\
\hline Electronic Hand dryers & & $x$ & & $x$ & & $x$ & & $x$ \\
\hline Hand hygiene posters near the Sink & & $x$ & $x$ & & $\times$ & & $\times$ & \\
\hline 1 alcohol hand rub for each bed & & $x$ & & $x$ & & $x$ & & $\times$ \\
\hline 1 alcohol hand rub for each personnel & & $x$ & & $x$ & & $x$ & & $x$ \\
\hline
\end{tabular}

a Abbreviation: PICU, pediatric intensive care unit.

Table 2. Observed Health Care Professional Contact With Patients in Different Departments a

\begin{tabular}{lccc}
\hline \multirow{2}{*}{ Department } & Total Contacts & \multicolumn{2}{c}{ RiskContact } \\
\cline { 3 - 4 } Emergency Ward & & $61(60.4)$ & $40(39.6)$ \\
Infectious Diseases Ward & 101 & $121(58.7)$ & $85(41.3)$ \\
PICU $^{\text {b }}$ & 206 & $60(58.8)$ & $42(41.2)$ \\
Gastroenterology Ward & 102 & $60(59.4)$ & $41(40.6)$ \\
Total & 101 & 302 & 208 \\
\hline
\end{tabular}

a Data are presented in No. (\%).

b Abbreviation: PICU, pediatric intensive care unit.

Table 3. HCP Hand Hygiene Behavior Observed in Different Departments of the Hospital ${ }^{a}$ b

\begin{tabular}{|c|c|c|c|c|}
\hline \multirow{2}{*}{$\begin{array}{l}\text { HCP Behavior Before Contact With } \\
\text { Patient }\end{array}$} & \multicolumn{4}{|c|}{ Number of Patient Contacts Observed in Different Departments } \\
\hline & Emergency $\operatorname{Room}(n=101)$ & Infectious Diseases $(\mathbf{n}=\mathbf{2 0 6})$ & PICU $(\mathbf{n}=102)$ & $\mathbf{G I}(\mathbf{n}=\mathbf{1 0 1})$ \\
\hline \multicolumn{5}{|l|}{ Hand washing } \\
\hline No & $101(100.0)$ & $206(100.0)$ & $102(100.0)$ & $101(100.0)$ \\
\hline Yes & 0 & 0 & 0 & 0 \\
\hline \multicolumn{5}{|l|}{ Using alcohol hand rub } \\
\hline No & $101(100.0)$ & $206(100.0)$ & $102(100.0)$ & $101(100.0)$ \\
\hline Yes & 0 & 0 & 0 & 0 \\
\hline \multicolumn{5}{|l|}{ Wearing disposable gloves } \\
\hline No & $87(86.1)$ & $161(78.2)$ & $82(80.4)$ & $82(81.2)$ \\
\hline Yes & $14(13.9)$ & $45(21.8)$ & $20(19.6)$ & $19(18.8)$ \\
\hline \multicolumn{5}{|l|}{ Wearing surgical gloves } \\
\hline No & $97(96.0)$ & $196(95.1)$ & $100(98.0)$ & $101(100.0)$ \\
\hline Yes & $4(4.0)$ & $10(4.9)$ & $2(2.0)$ & 0 \\
\hline \multicolumn{5}{|l|}{ Hand hygiene and wearing gloves } \\
\hline No & $101(100.0)$ & $206(100.0)$ & $102(100.0)$ & $101(100.0)$ \\
\hline Yes & 0 & 0 & 0 & 0 \\
\hline \multicolumn{5}{|l|}{ No action } \\
\hline No & $18(17.8)$ & $55(26.7)$ & $22(21.6)$ & $19(18.8)$ \\
\hline Yes & $83(82.2)$ & $151(73.3)$ & $80(78.4)$ & $82(81.2)$ \\
\hline
\end{tabular}

a Abbreviation: GI, gastrointestinal ward.

b Data are presented in No. (\%). 


\section{Discussion}

Our findings revealed that facilities available for the implementation of hand hygiene in different departments were far from optimal. Bed to sink ratio varied from 10:1 to $6: 1$ and bed to hand rub stations from $12: 1$ to $6: 1$; a study from India has shown no relationship between the number of sinks and hand hygiene behavior (9). All water faucets were hand-operated, but apart from the emergency department, paper towels were not available for drying wet hands. It is important to drying the hands completely after washing, as microorganisms are transferred in much larger numbers from wet hands than dry hands (6). Alcohol hand rubs were available in all the wards, but not for every patient, and isopropyl alcohol was used to top-up the containers. It has been demonstrated that $70 \%$ isopropanol or $70 \%$ ethanol is more effective against enveloped and non-enveloped viruses, than $4 \%$ chlorhexidine or other medicated soaps $(1,5)$. Studies have also shown that ethanol hand rub is superior to isopropanol for eradication of viruses (5).

Different methods have been advocated for monitoring hand hygiene compliance; we used direct observation which is considered as the 'Gold Standard' by most authors, although it may be prone to the 'Hawthorne effect' $(7,10,11)$. In the present study, compliance with hand hygiene was observed covertly by a pediatric resident, in order to make documentation as discreet as possible. It has previously been reported that if the HCPs are aware of being observed they may improve their adherence to hand hygiene by up to $55 \%(10,11)$. In our study, we observed a complete lack of adherence to the standard protocols for hand hygiene designed by the WHO, CDC, and other health care management authorities, at all levels of the HCP, physicians and nurses alike $(1,3,5,7)$.

Rates of hand hygiene compliance vary in different parts of the world; $19.6 \%$ and $20 \%$ compliance have been reported from an Italian and a Spanish teaching hospital, respectively $(12,13)$ approximately $32 \%$ from an internal medicine ward of a University Hospital in Turkey (14), and $34 \%$ in healthcare workers from a Pediatric Hospital in Rio de Janeiro in Brazil (15). A study from Southeast Iran regarding compliance of HCPs working in hemodialysis centers revealed a hand washing adherence rate of 58.7\%, while a study from Mashad, Iran, reported a hand washing rate in the health staff of a general hospital as $8.5 \%$ $(16,17)$. A systematic review on hand hygiene compliance by the HCP reports a median compliance rate of around $40 \%$, with the lowest rates found in ICU settings (18).

We are unable to account completely for the wide disparity found in the hand hygiene practices of HCP in our hospital and those reported from other parts of the world; a lack of awareness or lack of accessibility to AHR could be contributing factors. Only a few studies have dealt with the issues that determine HCP behavior (18). Some of the important factors that have been identified as having an impact on hand hygiene compliance in- clude; high work load, lack of accessibility to AHR, concerns about skin damage and lack of knowledge $(18,19)$. Moreover, it has been widely reported that compliance rates in physicians are consistently lower than nurses $(18,19)$. Although the HCP in our study did not comply with the standard recommendations for hand hygiene, they did use disposable or sterile surgical gloves for most situations that required hand hygiene implementation. Glove use is indicated in all situations involving blood exposure risk or body fluid exposure that may be contaminated with blood, and also when caring for patients with clostridium defficile diarrhea, but it does not obviate the necessity for hand hygiene $(1,4)$. It is important that hands are washed thoroughly or AHR used after the removal of gloves (4). In almost all of the observed contacts in our study, personnel did not wash their hands nor did they use AHR before donning disposable or sterile gloves and only four individuals performed hand hygiene after the gloves were removed. Inappropriate glove use, including the use of gloves for low risk procedures, failure to remove gloves and failure to perform hand hygiene after glove use has been reported from other studies as well $(20,21)$. Some researchers have named glove use as one of the risk factors for poor adherence to hand hygiene and an increase in the risk of cross-infection, but other studies have refuted this observation $(1,4,18,20,21)$. Not only does inappropriate use of gloves result in a waste of resources, but it may also cause an increase in the dissemination of pathogens between different locations, for example after putting on gloves the HCP may move from a clean to a dirty site and back to the clean site on the same patient, without changing the gloves $(4,18,20)$. It has also been noticed that HCP may wear gloves for selfprotection, rather than for the prevention of infection transmission between patients (13).

Our study had some limitations; as AHR dispensers were situated outside the 3 to 6-bedded rooms and the observers were inside the rooms, they may have missed the use of AHR before the HCP entered the room, and thus underestimated the level of conformity with standard hand hygiene guidelines. Nevertheless, it was observed that despite the presence of wash basins inside the rooms, the HCP did not wash their hands nor did they go out again to use the AHR between touching different patients.

Our study reveals a serious lack of adherence to standard hand hygiene protocols in all medical and nursing personnel, and highlights the need to educate HCP and increase awareness about the hazards of non-conformity with hand hygiene procedures. Facilities also need to be available for the implementation of hand hygiene in the different departments.

\section{Acknowledgements}

We would like to thank the staff of all the wards under observation for cooperating with us, without knowing what we were doing. 


\section{Authors' Contribution}

Dr Shiva: study design, literature search, drafting and review of the manuscript; Dr Kamali: acquisition of data, literature search, compiling the results; Drs Shirvani, Ghanaiei, Armin, Tabatabaiei and Fahimzad: teamwork in concept, design and critical review; Dr. Shamshiri: analysis of data and review; Dr. Karimi: Study concept and design, critical review, administrative support and supervision of the research.

\section{Financial Disclosure}

The authors do not have any conflict of interest. The authors do not stand to gain any monetary benefits through this research or its publication.

\section{Funding/Support}

No funding or financial support was granted for this research.

\section{References}

1. Clean Care is Safer Care Team. WHO guidelines on hand hygiene in health care. 2009. Available from: http://whqlibdoc.who.int/publ ications/2009/9789241597906eng.pdf.

2. Khodavaisy S, Nabili M, Davari B, Vahedi M. Evaluation of bacterial and fungal contamination in the health care workers' hands and rings in the intensive care unit.JPrev Med Hyg. 2011;52(4):2158.

3. Clean Care is Safer Care. WHO Five Moments for Hand Hygiene. 2010. Available from: www.who.int/gpsc/tools/Five_moments/ accessed.

4. Devnani M, Kumar R, Sharma RK, Gupta AK. A survey of handwashing facilities in the outpatient department of a tertiary care teaching hospital in India. J Infect Dev Ctries. 2011;5(2):114-8.

5. Boyce JM, Pittet D. Guideline for Hand Hygiene in Health-Care Settings. Recommendations of the Healthcare Infection Control Practices Advisory Committee and the HICPAC/SHEA/APIC/IDSA Hand Hygiene Task Force. Society for Healthcare Epidemiology of America/Association for Professionals in Infection Control/ Infectious Diseases Society of America. MMWR Recomm Rep. 2002;51(RR-16):1-4.

6. Sax H, Allegranzi B, Chraiti MN, Boyce J, Larson E, Pittet D. The
World Health Organization hand hygiene observation method. Am J Infect Control. 2009;37(10):827-34.

7. Hand Hygiene. Measuring Hand Hygiene Adherence: Overcoming the Challenges. 2009. Available from: http://www.jointcommission. org.

8. Fuller C, McAteer J, Slade R, Cookson B, Michie S, Stone S. Short Summary of Hand Hygiene Observation Tool (HHOT). London: Royal Free And University College; 2007. Available from: http://www. idrn.org/nosec.php.

9. Vernon MO, Trick WE, Welbel SF, Peterson BJ, Weinstein RA. Adherence with hand hygiene: does number of sinks matter? Infect Control Hosp Epidemiol. 2003;24(3):224-5.

10. Haas JP, Larson EL. Measurement of compliance with hand hygiene. J Hosp Infect. 2007;66(1):6-14.

11. Eckmanns T, Bessert J, Behnke M, Gastmeier P, Ruden H. Compliance with antiseptic hand rub use in intensive care units: the Hawthorne effect. Infect Control Hosp Epidemiol. 2006;27(9):931-4.

12. Pan A, Mondello P, Posfay-Barbe K, Catenazzi P, Grandi A, Lorenzotti S, et al. Hand hygiene and glove use behavior in an Italian hospital. Infect Control Hosp Epidemiol. 2007;28(9):1099-102.

13. Novoa AM, Pi-Sunyer T, Sala M, Molins E, Castells X. Evaluation of hand hygiene adherence in a tertiary hospital. Am J Infect Control. 2007;35(10):676-83.

14. Kuzu N, Ozer F, Aydemir S, Yalcin AN, Zencir M. Compliance with hand hygiene and glove use in a university-affiliated hospital. Infect Control Hosp Epidemiol. 2005;26(3):312-5.

15. Hofer CB, Abreu TF, Silva EG, Sepulveda CA, Gibara FA, Lopes NR, et al. Quality of hand hygiene in a pediatric hospital in Rio de Janeiro, Brazil. Infect Control Hosp Epidemiol. 2007;28(5):622-4.

16. Darvish Moghaddam S, Zahedi MJ, Dalili M, Shokoohi M. Compliance of healthcare professionals with safety measures for control of hepatitis viruses in hemodialysis centers: an experience from southeast iran. Hepatitis Res Treat. 2012;2012.

17. Naderi H, Sheybani F, Mostafavi I, Khosravi N. Compliance with hand hygiene and glove change in a general hospital, Mashhad, Iran: an observational study. Am J Infect Control. 2012;40(6):e221-3.

18. Erasmus V, Daha TJ, Brug H, Richardus JH, Behrendt MD, Vos MC, et al. Systematic review of studies on compliance with hand hygiene guidelines in hospital care. Infect Control Hosp Epidemiol. 2010;31(3):283-94.

19. Pittet D. Improving compliance with hand hygiene in hospitals. Infect Control Hosp Epidemiol. 2000;21(6):381-6.

20. Loveday HP, Lynam S, Singleton J, Wilson J. Clinical glove use: healthcare workers' actions and perceptions. J Hosp Infect. 2014;86(2):110-6.

21. Girou E, Chai SH, Oppein F, Legrand P, Ducellier D, Cizeau F, et al. Misuse of gloves: the foundation for poor compliance with hand hygiene and potential for microbial transmission? J Hosp Infect. 2004;57(2):162-9. 